\title{
Troussaeu's Syndrome in Association with Yolk Sac Tumour of Ovary
}

\author{
Papa Dasari', Maya G', Bhuvaneswari V2 \\ Dept of Obs/Gyn², Radio diagnosis ${ }^{3}$ \\ JIPMER, Puducherry, India
}

\begin{abstract}
:
Trousseau's syndrome is the spontaneous recurrent migratory thrombosis or arterial emboli due to non bacterial thrombotic endocarditis or both in a patient with underlying malignant neoplasm, which in this case of concern was the thrombosis in the Internal Jugular Vein (IJV) associated with yolk sac tumours of ovary is described.
\end{abstract}

Keywords: Spontaneous recurrent migratory thrombosis, Trousseau's syndrome, yolk sac ovarian tumour

\section{Introduction}

Trousseau's syndrome is defined as a spontaneous recurrent migratory thrombosis or arterial emboli due to non bacterial thrombotic endocarditis or both in a patient with underlying malignant neoplasm. Its association with ovarian malignancy was first reported by Womack and Castelenno in 1952 with papillary cystadenocarcinoma of ovary ${ }^{1}$. Subsequently few cases were reported to occur with mucinous tumors. Our literature search did not reveal any case report of Trousseau's syndrome with yolk sac tumours of ovary. To our knowledge only one case of Superior vena cava syndrome (SVC) has been reported with yolk sac tumor of testis ${ }^{2}$. Furthermore occurrence of thrombosis in Internal Jugular Vein (IJV) is rare and we found only one case report of IJV thrombosis with ovarian malignancy. Therefore we wish to document this case of IJV thrombosis in association with yolk sac tumour.

\section{Case}

A 21 year old unmarried lady presented with complaints of sudden progressive abdominal distension for 20 days associated with abdominal pain for one week. She gave history of loss of weight and loss of appetite for the preceding one month period. She had no past or family history suggestive of ovarian malignancy or prothrombotic disorders.
On physical examination she weighed $45 \mathrm{~kg}$, had mild pallor and did not have lymphadenopathy. Her cardiovascular and respiratory systems were normal. Abdominal examination revealed presence of free fluid and a 24 week size firm mobile mass arising from pelvis. On per vaginal examination, uterus was found to be of normal size with a firm mass felt through the fornices.

Transabdominal sonography showed ovarian mass measuring $14.8 \times 11.6 \mathrm{~cm}$ with multiple cystic and predominantly solid components with moderate ascites. Liver and both kidneys were normal. Her hematologic parameters were within normal limits with hemoglobin of $11 \mathrm{~g} \%$ and a bleeding time of 2 minutes and clotting time of 4 minutes. With regard to tumour markers, Alpha feto - protein was the only marker that was positive (3048 IU). HIV \& hepatitis $B$ surface antigen were negative.

At laparotomy 3 litres of straw colored ascitic fluid was present and the right ovary was replaced by a congested bosselated tumour of $18 \times 10 \mathrm{~cm}$. Left ovary appeared to be normal. Right saphingo-ovariotomy with left ovarian biopsy was done. Omentectomy was also done. Mesenteric lymph nodes were found to be enlarged: $0.5 \mathrm{~cm} \times 0.5 \mathrm{~cm}$. The cut section of the tumour was

Correspondence

Dr. Papa Dasari MD

Prof Dept of Obs/Gyn,

JIPMER, Puducherry, India

Phone: 9442566883

Email:dasaripapa@gmail.com 
predominantly solid with few cystic areas filled with hemorrhagic material. Solid areas were greyish white to greyish yellow in colour and there was no normal ovarian tissue. Histopathological report revealed pure yolk sac tumor of right ovary with characteristic Shiller- Duval bodies with reticular microcytic pattern. Left ovarian biopsy revealed normal tissue. Omentum was free of tumor.

On the ninth post operative day, patient developed painful swelling on right side of the neck lateral to the sternocleido mastoid muscle. All peripheral pulses and carotid pulses were palpable. Doppler ultrasonography showed dilated, non compressible right internal jugular vein with heterogeneous echogenicity extending proximally to right brachiocephalic vein. Similar echogenicity was observed in right subclavian vein. Distally it was seen to extend till external jugular vein branch. The diagnosis of right internal jugular vein thrombosis with right subclavian vein thrombosis was made and she was anticoagulated with inj. heparin 5000 IU every 8 hrs subcutaneously. Her prothrombin time was 20 " with INR of 1 . Tablet Nicoumalone $4 \mathrm{mg}$ and aspirin $75 \mathrm{mg}$ per day were started from the third day. CT thorax (Fig 1,2) showed luminal hypodensities in right internal jugular vein for a length of $7 \mathrm{~cm}$ and in right subclavian vein suggesting thrombosis. Superior vena cava was free. Other structures of chest were normal. Neck swelling subsided in 2 days. Inj. Heparin was stopped after 4 days as her INR was 2.2 and she was started on oral anticoagulation .Repeat Doppler after 2 weeks did not reveal any resolution of thrombus and her INR was 1.83. She was restarted on Heparin which was continued along with oral anticoagulant for 3 weeks. INR at this time was 2.41 and heparin was stopped and she was started on first cycle of chemotherapy with BEP regimen (Bleomycin, Etoposide, and Cisplatin) which she tolerated without any side-effects. All other haematological parameters were normal. Repeat Doppler examination (After 3 weeks of anticoagulants) showed resolution of thrombus in subclavian vein. CT abdomen and pelvis were normal without any evidence of residual tumour masses and her INR was maintained at 1.5. Alpha fetoprotein was 9IU/1 after 2 cycles of chemotherapy and was $3 \mathrm{IU} / 1$ after 6 cycles. She completed 6 cycles of chemotherapy, the only significant side effect being alopecia. One month after chemotherapy she presented with difficulty in breathing and chest pain. X-Ray chest P/A showed reticular pattern suggestive of bleomycin induced fibrosis. CT thorax showed presence of collaterals (Fig 3) and lung fields showed fibrosis especially on right side with minimal pneumothorax (Fig 4,5). She was treated with steroids and bronchodilators for one month and advised to continue oral anticoagulants. She is now on regular follow up and is free of symptoms since 2 months.

\section{Comment}

Internal Jugular thrombosis was first described in 1912 by Long as a complication of peritonsillar abscess. It is an uncommon and fatal condition and usually occurs due to trauma by catheterisation or repeated injections in to the vein by intravenous drug users. Other causes include malignancy, ovarian hyperstimulation syndrome and coagulation disorders ${ }^{3}$. It can rarely be the first clinical diagnosis when a patient presents with a neck swelling as reported by Lam WM and colleagues in a case of metastatic adenocarcinoma of unknown primary ${ }^{4}$. The present case had malignancy in the form of yolk sac tumour of ovary. In the case reported by Ball and colleagues, the patient had advanced ovarian malignancy, coagulopathy and insertion of a central line in to the internal jugular vein and hence the cause of internal jugular vein thrombosis was not clear ${ }^{3}$.

Internal Jugular vein thrombosis refers to an intraluminal thrombus occurring anywhere from intracranial IJ vein to the junction of the IJ and Subclavian vein to form the brachiocephalic vein ${ }^{5}$. In the present case the thrombus extended from right Subclavian vein to the junction of external and internal jugular vein. It is important to know this to understand the prognosis. The rates of pulmonary embolism are $0.5 \%$ for isolated internal jugular thrombosis and $2.4 \%$ for combined IJ and subclavian / axillary vein thrombosis. Mortality rates at 1, 3 and 12 months have been reported to be $14 \%, 33 \%$ and $42 \%$ respectively ${ }^{5}$. Prolonged anticoagulation is necessary for these cases to prevent complications and recurrences. Callender $\mathrm{N}$ and Rapaport colleagues concluded from their 4 case reports that the patients with Trousseau's syndrome have persistent low grade intravascular coagulation and anticoagulation should be continued indefinitely. They proposed that Trousseau's syndrome results due to the expression of tissue factor on the tumour cells and also due to the exposure of cells or vesicles shed from the tumour to the circulating blood directly or indirectly ${ }^{6}$. This is further strengthened by the observation made by Evans Jeffry and colleagues that breakthrough thromboembolic events still occurring despite what would otherwise be considered as satisfactory levels of anticoagulation (INR). An INR above the normally recommended therapeutic range of 2 to 4.5 may be necessary to prevent further thromboembolic episodes ${ }^{7}$. The INR was kept at 2.4 in this case when chemotherapy was administered.

Of all the modalities to diagnose Internal Jugular vein thrombosis, USG, CT and MRI were considered to be better because of their non-invasive nature and as they can also give additional information about 


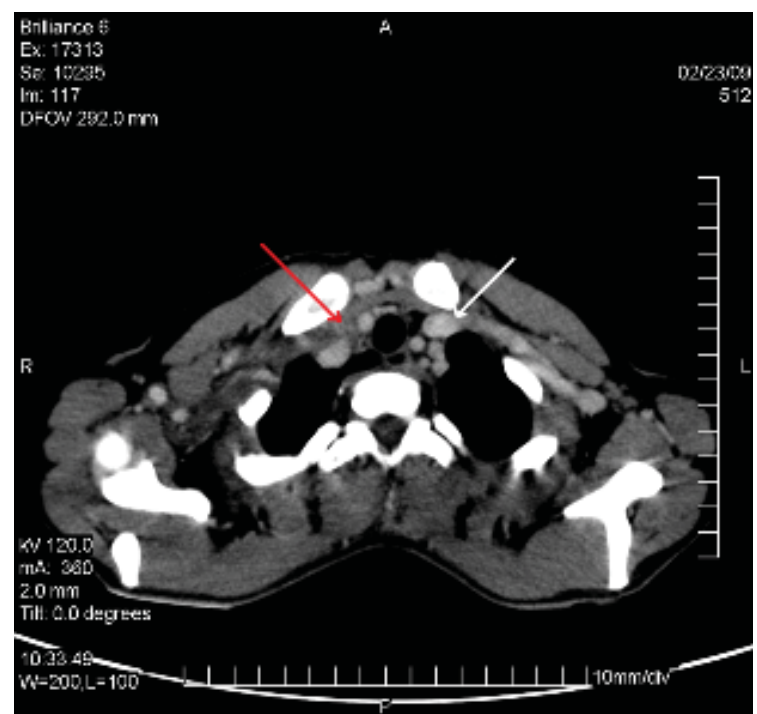

Fig 1. Post-contrst CT axial image at the thoracic inlet Red arrow shows the filling defect in the right subclavian vein and the white arrow points to the normal left subclavian vein.

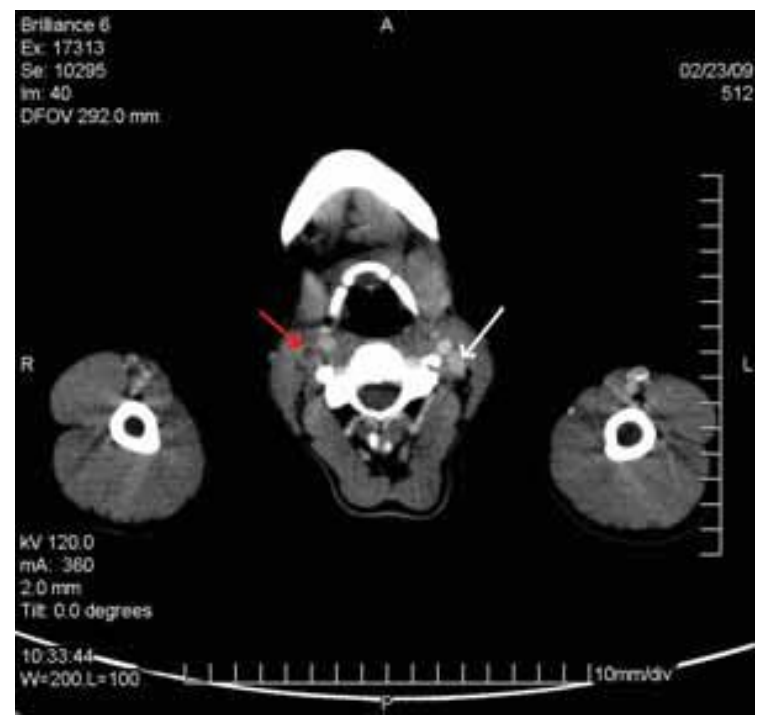

Fig 2. Post-contrast CT axial image at the level of hyoid bone Partially thrombosed right internal jugular vein is shown by red arrow. Normal filling of left internal jugular vein is shown by white arrow.

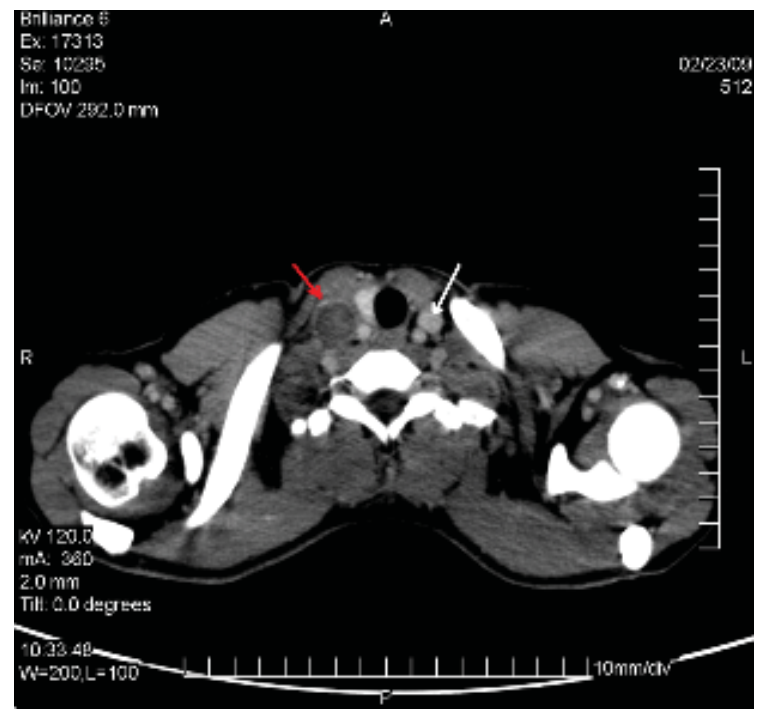

Fig 3. Post-contrast CT axial image at the level of thyroid Red arrow points to the dilated thrombus filled right Internal jugular vein Normal left IJV is shown by white arrow.

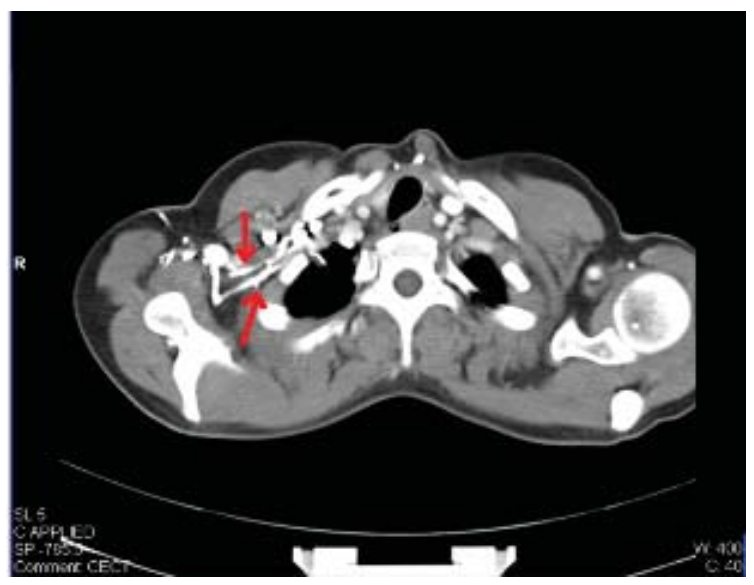

Fig 4. Post-contrast CT axial image at the level of thoracic inlet after 7 months Red arrows show the collateral veins

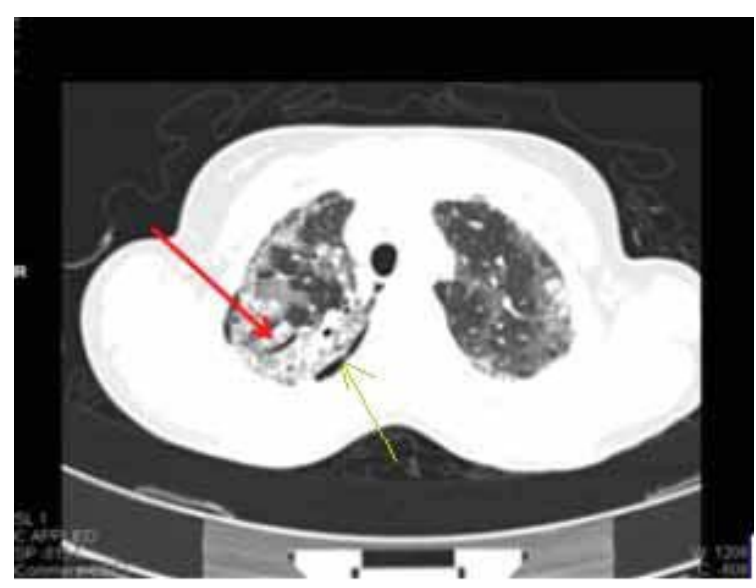

Fig 5. Post-contrast CT thorax Red arrow-Right side of lung shows reticular fibrosis and green arrow points to pneumothorax 
the cause such as extrinsic compression or invasion by malignancy of surrounding structures. CT thorax was undertaken in this case to confirm the diagnosis as well as to rule out any extrinsic cause. Real time USG with Doppler is the recommended imaging modality as it is useful for diagnosis as well as for monitoring the progress of thrombus non-invasively ${ }^{4}$. We also agree with this as this is cost-effective and helped in monitoring the extension / resolution of thrombus.

\section{Conclusion}

This rare and fatal condition of Trousseau's syndrome, illustrating the occurrence of internal jugular vein thrombosis in association with yolk-sac tumour of the ovary, successfully treated by prompt anticoagulation keeping INR between 2 to 4 thereafter followed by safely administered chemotherapy, sets an example that such a case can be managed without causing the mortality if recognised early.

\section{References}

1. Womack WS, Castellano CJ. Migratory thrombophlebitis in association with ovarian carcinoma. Am J Obstet Gynecol 1952; 63: 467692.

2. Ingram L, Rivera GK, David NS. Superior vena cava syndrome associated with childhood malignancy: An analysis of 24 cases, medical and Paediatric Oncology 2006; 18 (6):476-481.

3. Ball E, Morris -Stiff G, Coxon M and Lewis MH. Internal jugular vein thrombosis in a warfarinised patient: a case report. Journal of Medical Case Reports 2007; 1: 184-186.

4. Law HM, Ahuja AT, Mak Co, Metrewelic. Spontaneous Internal Jugular Vein thrombosis and metastatic adenocarcinoma of unknown primary. HKMJ 1995; 1: 258-260.

5. Muller DK, Dacey MJ. Internal Jugular vein Thrombosis 2009; http://emedicine. medscape.com /article/461577-overview

6. Callander N, Rapaport SI. Trousseau's Syndrome. West J Med 1993; 158: 364-371.

7. Jeffry Evans TR, Mansi JL, Bevan DH. Trousseau's syndrome in association with ovarian carcinoma. Cancer 1996; 77(12):25442549. 